\title{
LA GRIETA EN EL LENGUAJE (BIOGRAFÍA DE UNA ILUSTRE POÉTICA DEL AZAR) ${ }^{1}$
}

\author{
Melvin Campos Ocampo
}

\begin{abstract}
RESUMEN
El presente estudio indaga en los planteamientos teóricos acerca del lenguaje, la escritura y la literatura que se encuentran en dos textos del escritor costarricense Alí Víquez Jiménez: "Labores o desdichas del profesor Friedrich Hinkelmaier" y "Un texto roto". En ellos se presenta una visión de la literatura como un capricho editorial; una imagen del ser humano como un simple mono parlante y una exposición teórica sobre el lenguaje, el saber y la falta.
\end{abstract}

Palabras clave: Literatura costarricense, Alí Víquez Jiménez, Biografías de hombres ilustres.

\begin{abstract}
The following article explore the theoretical proposition of language, writing and literature that appear in two short stories by Costa Rican writer, Alí Víquez Jiménez: "Labores o desdichas del profesor Friedrich Hinkelmaier" and "Un texto roto". These texts propose to understand literature as an editorial whim, human being as mere talking ape, and offer a particular meditation on language, knowledge and lack.

Key words: Costa Rican Literature, Alí Víquez Jiménez, Biografías de hombres ilustres.
\end{abstract}

Para Alí Víquez quien, ahora que es padre, termina una vida y comienza otra.

...un observador omnisciente podría redactar un número indefinido, y casi infinito, de biografías de un hombre, que destacaran hechos independientes y de las que tendríamos que leer muchas antes de comprender que el protagonista es él mismo. Jorge Luis Borges Sobre el Vathek de William Beckford

\section{Prae textus (Digresión justificadora)}

Todo texto literario es una concreción, al menos, de una poética particular. En la época a.B. (antes de Barthes), se consideraba que era "la poética de su autor"; pero en estos tiempos menos creyentes (y por ello, acaso, más desamparados, pero también más literarios), sabemos

Melvin Campos Ocampo. Licenciado en Filología Española por la Universidad de Costa Rica. Profesor de la Universidad de Costa Rica y de la Universidad Estatal a Distancia. San Pedro, San José, Costa Rica.

Correo electrónico: mcampos@ticomedia.com 
que cada autor es sólo un personaje más de ese gran texto ficticio y verosímil, lleno de versiones, que llamamos historia.

Cada texto, entonces, posee su poética, es una concreción de una poética determinada. En algunos casos es explícita y en otros oculta, pero todos los textos comportan, al menos, una definición aproximativa del lenguaje y de lo literario. Dos textos, atribuidos al nombre de Alí Víquez, "Labores o desdichas del profesor Friedrich Hinkelmaier" y "Un texto roto" (incluidos ambos en la colección Biografías de hombres ilustres), me han interrogado en ese sentido. Y como todo texto vive únicamente mientras es leído, he optado por hacer una biografía de estos dos. Los conocí durante toda su vida: desde que abrieron sus páginas ante mí y me dijeron sus primeras palabras, hasta que suavemente y con duelo, tuve que presenciar su punto final.

Borges, inquietante como siempre, sugiere que existen miles de textos posibles de una sola vida, según los hechos que se seleccione narrar. Yo me centraré sólo en un aspecto de sus vidas: rastrear los postulados acerca de la literatura y del lenguaje que ellos exponen, en otras palabras, su poética. Mi búsqueda sigue este camino.

\section{2. Ética / Poética / Política (El proyecto Hinkelmaier)}

El relato que abre el volumen es "Labores o desdichas del profesor Friedrich Hinkelmaier" y con él abriremos nuestra reflexión. El texto refiere la relación entre el narrador y el profesor Hinkelmaier, quien tiene en su casa dos habitaciones misteriosas: en una encierra un grupo de monos y en otra guarda una colección de microfilmes. Me permitiré separar el texto en dos partes, una para cada cuarto, y empezaré por la segunda, pues ésta plantea un concepto más extendido en la teoría literaria contemporánea.

El contenido de la habitación misteriosa número dos es, como dijimos, una colección de microfilmes, los cuales registran todos los textos rechazados por una gran cantidad de editoriales del mundo.

Nace, entonces, una pregunta obvia: ¿para qué semejante trabajo si -a juzgar por el rechazo editorial- los textos han de ser mediocres?

Hinkelmaier responde con una anécdota: siendo joven, recibió un manuscrito literalmente nauseabundo; él promovió su rechazo y sugirió que aquella porquería se llamara $L a$ náusea... (Víquez 2002: 9). El escritor se convirtió en Jean-Paul Sartre y el libro en un hito filosófico. Hinkelmaier se da cuenta de su error y emprende su cruzada para evitar:

\footnotetext{
...las equivocaciones increíbles que registra la historia de los editores: [...] los casos de decenas de imprescindibles autores literarios y científicos, que habían penado consiguiendo la acogida editorial de sus obras maestras (Víquez 2002: 10).
}

Constatando la teoría de Hinkelmaier, la historia fantástica de la literatura cuenta una leyenda acerca de cómo la gran Virginia Woolf recibió, de un joven irlandés, el manuscrito de una novela que no contaba nada: cientos de páginas y no pasaba nada. Por supuesto, doña Virginia lo rechazó. El joven era James Joyce y el texto se intitulaba Ulises $^{2}$.

Con esta preocupación, Hinkelmaier decide rescatar todo texto rechazado por alguna editorial del mundo. Y su proyecto ha demostrado ser valioso, pues ya en varias ocasiones un texto de su biblioteca de excluidos ha sido 
...descubierto por la crítica y le hacen ediciones multitudinarias, por lo que debo sacarlo abruptamente de la colección; no me duele: eso sólo le da más validez al proyecto (Víquez 2002: 11).

Así, Hinkelmaier guarda todo, pues cualquiera de sus textos puede llegar a ser una obra maestra. Todo depende del tiempo y de lo que las editoriales dictaminen.

Revisemos dos premisas que se extraen de las reflexiones del profesor.

En primer lugar, aparece una respuesta materialista a la pregunta clave de la teoría literaria, la famosa “¿qué es la literatura?”. Según lo propuesto por Hinkelmaier, la literatura es un hueco, un vacío, una nada que la sociedad llena con cualquier texto, escogido según gustos y conocimientos personales de los editores (y, por supuesto, según las particularidades psíquicas y los condicionamientos ideológicos a los que se encuentren sujetos en su contexto histórico). No existe algo intrínseco a los textos que los determine como literarios, no hay tal cosa como un valor literario universal, no se trata de una característica óntica. Habría que considerar, entonces, que lo literario es variable, fluctúa en el tiempo y en el espacio según cambien los sujetos, las sociedades y los gustos editoriales.

Por otra parte, de la reflexión de Hinkelmaier también se extrae que quien dictamina lo literario no es una entidad tan difusa como "la sociedad"; y, en una suerte de epifanía sociocrítica, el profesor ve el poder de las editoriales sobre la literatura: son ellas las que definen lo literario y se descubre, así, la existencia de los instituyentes literarios.

En efecto, para Hinkelmaier, no es la sociedad quien decide qué meter en ese hueco que es la literatura, sino una especie de oligarquía editorial, a modo de los Superiores Desconocidos de Umberto Eco. Esta cofradía de editoriales tomaría la decisión de qué es lo literario y qué no ${ }^{3}$.

El "descubrimiento" de Hinkelmaier no es desconocido en el mundo filológico, pero repensémoslo un momento. Tanto para filólogos como para cualquier laico amante de la literatura, el asunto es trágico: sólo podemos leer caprichos editoriales. Todo lo que nos ha llegado, llega y llegará es una selección de libros realizada por un grupo de editoriales que, lejos de valorar un texto por sus reflexiones filosóficas, literarias o sociales, utilizan como única medida las posibilidades de venta que tenga.

D.W. Fokkema (1982: 111-2) sugería un camino para soliviantar esta angustia. Como justificación social del estudio de la literatura y para incluir al filólogo en este aparato editorial, proponía una suerte de mercadotecnia literaria. Según él, a partir de la teoría de la recepción, se debía especular el gusto de los lectores para que las editoriales, los medios de comunicación y los gobiernos pudieran saber qué publicar. Desgraciadamente, la posición de Fokkema -muy poco ética, en mi opinión-, lejos de solucionar el problema, hace al estudioso de la literatura cómplice de él.

Contrario a la propuesta de Fokkema, surge el proyecto de Hinkelmaier: el anciano no se contenta con haber vislumbrado el fenómeno, sino que se propone hacer algo para combatirlo. La poética que plantea Hinkelmaier no es sólo una develación de los instituyentes literarios, se trata de un planteamiento ético en relación con esos instituyentes: recopilarlo todo, darle voz a los marginados, rescatar la palabra del silenciado.

Así, como el trabajo del profesor busca dar refugio a todo lo rechazado por las editoriales, es factible extrapolar que, para él, lo olvidado en algún momento tendrá cabida dentro del universo literario conocido. En otras palabras, todo texto que, en opinión de las editoriales, debe ser olvidado, relegado, excluido del sistema textual literario, para Hinkelmaier eventualmente 
tendrá un valor dentro del sistema. El proyecto Hinkelmaier es, entonces, una alternativa, una contestación al olvido editorial de que es objeto una gran cantidad de textos.

Este proyecto se nos revela desde el título, "Labores o desdichas del profesor Friedrich Hinkelmaier”. En él se establece una relación diádica entre los términos 'labor' y 'desdicha'. En español, la conjunción 'o' puede establecer tanto una oposición como una igualdad entre los términos que reúne ${ }^{4}$, por lo cual podemos entender que las labores de Hinkelmaier también son desdichas. El mismo profesor se define como "el más sufrido de los editores" (Víquez 2002: 2).

Nótese que la autodefinición de Hinkelmaier, al ser un editor, vincula necesariamente su tragedia con la palabra, con un trabajo sobre la palabra. Esta coincidencia se explica porque este desdichado sufre de una des/dicha: su tragedia es una palabra no dicha. Es un editor que sufre por la palabra no dicha, por una palabra silenciada, oculta, relegada al olvido; y su proyecto busca erradicar eso.

Hinkelmaier es claramente bajtiniano, pues expone una teoría que rescata las prácticas discursivas marginadas, que revalora la presencia de voces alternas en la palabra social, que considera que, aunque los instituyentes traten de silenciarlas, esas voces están presentes en la palabra, actúan en ella y deben ser escuchadas. Propuestas todas que pertenecen al pensamiento de Mijail Bajtín y su concepto de palabra dialógica (Zavala 1991: 188-9).

El propio narrador se da cuenta de la búsqueda ética del profesor y no vacila en atribuirle la fundación de los sistemas de democratización textual, como la Internet (Víquez 2002: 13), ni en especular la forma última a la que debería llegar el proyecto Hinkelmaier:

Acaso lo que más convenga a la humanidad aún esté lejos: la muerte de las obras canónicas y la profusión del libro en absoluta libertad (Víquez 2002: 15).

Utopía socialista y democrática, en que el narrador ve la máxima expresión del proyecto Hinkelmaier. El profesor nos expone, entonces, una visión bastante materialista y sociocrítica del fenómeno literario: la literatura es un hueco que se llena con lo que las editoriales deciden. Por ello propone que esta situación debe ser combatida mediante un camino marxista, revolucionario, ético y activista a favor de la palabra silenciada, de los marginados en los circuitos literarios de distribución y consumo.

Pasemos, ahora, al cuarto de los monos.

\section{Simius Loquens o la poética del azar (EI experimento Hinkelmaier)}

Al iniciar el relato, cuando el narrador conoce a Hinkelmaier, se da cuenta de que el anciano tiene bajo su cuidado un grupo de monos a quienes ha bautizado con nombres de escritores: Cervantes, Shakespeare, Goethe, Homero, Dante y Dostoievski. En su primera visita a la casa del profesor, se siente intrigado por la primera habitación que ve, pues la encuentra cerrada, escucha unos extraños golpeteos que vienen de adentro y sabe que ahí están los primates. Luego, tiene la oportunidad de leer tres de los textos repudiados por las editoriales (que Hinkelmaier guarda en la segunda estancia) y asume que debe existir una relación entre ambos cuartos. Se le ocurre, entonces, una idea inquietante: los simios escribieron los textos. 
Hinkelmaier trataba de verificar la hipótesis de que un millón de monos tecleando desordenadamente durante un millón de años produciría una profusa cantidad de basura, mas también el portentoso Hamlet (Víquez 2002: 6).

Se precipita al interior de la habitación, únicamente para confirmar sus sospechas: Dante está tecleando en una máquina de escribir. El narrador arranca la página, pero en ésta sólo hay incoherencias ${ }^{5}$. Poco después, Hinkelmaier revela que no hay relación alguna entre los monos y los textos; el relato entonces toma el camino que exploramos en la parte anterior de nuestra reflexión.

Ignoraremos el hecho de que el relato derive hacia otra historia, por dos razones. En primer lugar, notemos un detalle: si Hinkelmaier no estaba realizando el experimento, ¿por qué había una hoja en la máquina que estaba usando Dante? Un mono no tiene la habilidad motriz para poner papel en el rollo de la máquina, lo cual implica que Hinkelmaier la puso y se la dio al primate amante de Beatriz. Hinkelmaier sí está realizando el experimento, pero le dice al narrador lo contrario. ¿Por qué el profesor desea ocultar su indagación? Tal vez por las implicaciones que tiene, las cuales veremos más adelante.

En segundo lugar, el hecho de que el relato se desvíe no elimina la propuesta teórica que ya se hizo ni deja al lector más tranquilo. Por el contrario, la evasión -tanto de Hinkelmaier como del narrador- fomenta la incertidumbre que deja la primera sospecha, la inquietud se queda, la pregunta persiste: ¿sería posible que un mono, con suficiente tiempo, lograra escribir algo coherente?

Es en esta duda donde aparece el primer postulado de esta parte: el experimento Hinkelmaier es, en teoría, correcto: los monos lograrán escribir Hamlet. La certeza inicial del narrador lo confirma:

\footnotetext{
¡Dios mío, es verdad! Los monos han hecho los textos de los microfilmes [...] Se hallaría tal vez, entre tantísimos microfilmes, uno en el que el triste simio que estaba frente a mí [Dante] había escrito, punto por punto, la comedia que el otro triste Dante compuso pensándose inspirado por el amor a Beatriz (Víquez 2002: 6).
}

El único trabajo que tendrán que realizar los primates es combinar azarosamente los caracteres de la máquina -letras, números, signos de puntuación y demás- para que, en algún momento, por obra de la casualidad, el príncipe de Dinamarca tome forma en sus páginas.

Aquí sobreviene la inquietud del narrador (y del lector con él) y, ante la certeza de que los simios han escrito los libros mediocres y pueden, por consiguiente, escribir el Quijote, el propio narrador expone el postulado teórico literario:

El genio literario no existe; sólo existe la obra de la casualidad (Víquez 2002: 6).

En efecto, Fausto, Hamlet, el Quijote, La divina comedia, Los hermanos Karamazov y La odisea son simplemente obras del azar, los grandes hitos de la literatura son un mero producto de la casualidad, burdas concreciones de la fortuna.

¿Absurdo? Pensémoslo un momento: millones de seres humanos escribiendo desde Sumeria hasta Japón, desde hace 5.000 años hasta hoy jueves 27 de mayo de 2004, infinita cantidad de palabras pronunciadas, trillones de páginas escritas en miles de caracteres de cientos de idiomas... Es comprensible que entre tantos pliegos, al menos, hayan salido seis libros 
de gran talla. Y si lo puede hacer el ser humano con sólo combinar letras, ¿por qué no un mono en igualdad de condiciones?

La dificultad que debe enfrentar Pierre Menard no es la de escribir el Quijote, sino la de repetirlo. Ya el Quijote se dio; concretarlo de nuevo sería forzar la casualidad.

Deseo indagar en otro aspecto relacionado con los monos de la escritura. El hecho de que los primates reciban nombres de escritores construye una cierta imagen de la literatura.

Es necesario acotar que el paralelismo entre los simios y sus correspondientes escritores va más allá del nombre: Cervantes y Shakespeare mueren el mismo día, "con pocas horas de diferencia"6 (Víquez 2002: 8); a Dante se le atribuye la Divina Comedia, recorre los infiernos y luego va al Paraíso; ante la muerte, Goethe tiene "una mirada solemne que nos demostraba una comprensión superior de aquella tragedia" (Víquez 2002: 8); Homero ignora su entorno como si estuviera ciego; y Dostoievski "era el de carácter más explosivo" (Víquez 2002: 8). Por último, el narrador regala otro mono a Hinkelmaier, quien lo bautiza como Víctor Hugo y lo define como un "primate romántico" (Víquez 2002: 12). Inclusive, el profesor califica al fenecido Dante como un "artista” (Víquez 2002: 8).

Señalemos un dato curioso: para muchas culturas, el simio es una figura de sabiduría. En el Tibet, por ejemplo, el Rey Mono acompaña al sabio Hiuan-Tsang a encontrar los libros sagrados del budismo y logra alcanzar el estado de Buddha. Además, en China, los tres conocidos simios del Jingoro - uno de los cuales no dice maldad, otro no escucha maldad y el otro no ve maldad- son paradigma del sabio incorruptible. Pero resulta muy sugerente la interpretación que dan las culturas mesoamericanas al simio: el signo zodiacal del mono era marca apolínea y sus protegidos eran artistas, cantores, escultores y escritores (Chevalier 1999: 718-9).

Coincidente con la visión mesoamericana, los simios de Hinkelmaier son escritores. De esta metáfora podemos extraer un visión contundente en relación con los grandes nombres de las Bellas Letras: este paralelismo implica una propuesta que coincide con la casi igualdad entre hombre y mono que ya mencionamos. Los grandes escritores, los Autores, los pilares de la literatura, no son más que simples monos con palabras, lugares donde el azar concreta una porción de lenguaje. Crimen y castigo, Werther y La tempestad no son más que casualidades. El genio literario no existe.

Así, Hinkelmaier, barthesiano hasta el extremo, no contento con demoler el concepto de autor (Barthes 1987: 71), lo convierte en un simple mono con letras, sin la fascinación que Paz podía sentir por él. Todo lo consabido de la literatura: los grandes autores, las grandes obras, todo depende del azar. No hay certeza, no hay absolutos literarios, el autor ha muerto y el escritor no es más que un vehículo del azar.

No obstante, reflexionemos un poco más. Si Goethe y Dostoievski no eran más que monos de la escritura, ¿dónde quedamos los demás seres humanos? ¿Es acaso que somos otra cosa que simios parlantes, tristes primates del lenguaje, monos gramáticos? ¿Todas las horas que dediqué yo a presionar teclas, cortar y pegar, a cambiar de lugar palabras y razonamientos, eso me diferencia en algo del Dante de Hinkelmaier? ¿Acaso por creer que sabemos lo que decimos nos vamos a sentir superiores? ¿No somos solamente monos ensayando ante una página en blanco? ¿Qué nos diferencia de ellos?

Según las ideas de Hinkelmaier, los propios seres humanos no somos más que vehículos del lenguaje; él decide si mi discurso tendrá sentido en español o si mi audiencia creerá que estoy hablando en maorí, en francés del siglo XXVI o en lenguas. Somos instrumentos del lenguaje. 
Quisiera anotar un par de ideas para concluir con Hinkelmaier. En primer lugar, el texto que escribe el simio Dante no significa nada en español, lo cual no implica que no signifique nada en alguna otra lengua. Y aunque no signifique nada en nuestras lenguas contemporáneas, eso tampoco impide que, en alguna lengua pasada o futura o extraterrestre, esa hoja digitada por el simio pueda ser, por ejemplo, una traducción de La canción desesperada.

Además, deseo colaborar con la inquietud que produce Hinkelmaier al lector: la hoja que escribe el Dante simio no es del todo incoherente. El narrador no se da cuenta de ello (¿o no quiere hacerlo?) y la atención del lector es desviada para que ignore este hecho. Sin embargo, el mono escribió “tapantî” (Víquez 2002: 7; ver nota 4). Ello nos demuestra, justamente, que la hipótesis de Hinkelmaier se empieza a comprobar. En este momento, si tuviera efectos de sonido, debería retumbar la obertura de Así hablaba Zarathustra.

\section{La grieta del texto (Algo acicatea...)}

El otro relato que nos interesa es el que lleva por título "Un texto roto". En forma de ensayo expositivo, se trata de una especulación acerca de una fantástica doctrina -práctica y filosófica- que reflexiona sobre los rompecabezas. El texto lleva ese título pues el final se ha perdido y unos supuestos editores intentan referir lo desaparecido, pero aún a ellos les falla la memoria.

Se trata entonces de un texto incompleto, al que le falta una parte. Podemos, entonces, entender el texto mismo como un rompecabezas al que se le ha perdido una pieza. Los editores concuerdan:

Ironía del destino la desaparición de un trozo de papel [perteneciente al texto mismo] que precisamente nos habla de cómo juntar las piezas de los rompecabezas (Víquez 2002: 87).

En efecto, el texto es un rompecabezas al que le falta una pieza. Partiendo de esta idea, es válido invertir la ecuación para interpretar, en el texto propiamente, la figura del rompecabezas como metáfora del texto y entender, a su vez, todos sus postulados en relación con los rompecabezas como metáforas de postulados acerca del lenguaje.

Si se me concede esta gracia, y se me permite interpretar los rompecabezas como textos, procederé a correlacionar las piezas con significantes, la ilustración que sirve de referencia a todo rompecabezas, con el referente y el fragmento de ilustración impreso en cada pieza, con el significado. Adelanto que, como veremos, la concepción de lenguaje elaborada por "Un texto roto" es de tendencia psicoanalítica, particularmente lacaniana.

El relato refiere, en primera instancia, cómo en el siglo XVIII nace el rompecabezas tal y como lo conocemos hoy, sólo que con una muy reducida cantidad de piezas, de 100 a 200, y viene acompañado por una ilustración que servía al armador de referente:

... con toda simpleza, el armador únicamente se afanaba en ordenar las piezas para reproducir una ilustración conocida de antemano (Víquez 2002: 86).

Este tipo de rompecabezas/texto primario sugiere una visión tradicional del lenguaje, un concepto referencialista a partir del cual se asume una equivalencia entre el texto (el rompecabezas) y el referente (la ilustración). Esta idea de igualdad entre los dos términos se retoma en 
la "Nota de los editores" cuando se propone una relación entre los rompecabezas y la realidad (Víquez 2002: 94). El propio narrador considera que esta equivalencia es una:

Doble ingenuidad; no sólo se afirmaba la identidad entre el rompecabezas y la ilustración adjunta, también se pensaba que los distintos modelos de esta ilustración eran intercambiables entre sí (Víquez 2002: 88).

Dicha relación de igualdad entre significante y referente es, para la teoría psicoanalítica del orden de la identificación diádica, propia del registro imaginario. Es ésta la etapa segunda del desarrollo, posterior al momento real y previa a la introducción de lo simbólico (Baudes 1995: 28). En la etapa imaginaria se establecen equivalencias entre Yo y Tú, que es la relación del narcisismo primario -identidad entre el rompecabezas y la ilustración-, y entre Yo y Yo, que es la fase del espejo de identificación unitaria del niño -modelos de rompecabezas intercambiables o dobles.

Ahora bien, en el siglo XIX se da un fenómeno peculiar: aparecen los rompecabezas cuya imagen referente era falsa. Para engañar a los armadores se introducen pequeñas diferencias en la ilustración con respecto al rompecabezas, las cuales desmienten la relación de equivalencia entre rompecabezas e ilustración (Víquez 2002: 86). Con ello se demuestra que el significante ya no corresponde al referente, ya no hay una identificación entre ambos. El referente es falso; el significante está vacío. Hemos pasado del registro imaginario al simbólico.

\footnotetext{
... surge la necesidad de preguntarse cómo difiere cada modelo de su ilustración y, por ende, el deseo de conocimiento. Mientras se creyó con ingenuidad que el rompecabezas era igual que la ilustración, nada había para preguntarse. Cuando se supo con madurez que cada rompecabezas armado era distinto de la ilustración a partir de la cual se había creado, comenzó a gestarse la sabiduría (Víquez 2002: 87).
}

De manera que, cuando se introduce una diferencia, se pierde la relación imaginaria de equivalencia entre significante y referente. Con esa diferencia se pierde algo - ¿una pieza del rompecabezas?, ¿una página del texto?-, se reconoce que hay algo que falta y nace la lucha por encontrarlo. En ese momento se funda un deseo, el deseo por encontrar eso que falta, y en ese momento también se funda un saber.

Posteriormente, los rompecabezas comienzan a hacerse en color y, aunque algunos pesimistas consideran esto como un retroceso porque "la gente pensaría que los rompecabezas reproducen la realidad" (Víquez 2002: 91), lo que pasa es que sobrevienen los rompecabezas no figurativos que son puro color, sin intención de representar ningún elemento de la realidad.

Esto acentúa la independencia que tiene el significante del referente: el significante puede decir cosas que no tienen referente alguno. Pero el asunto se extrema aún más cuando surgen los rompecabezas de un solo color. Anteriormente, una pieza se ubicaba en el rompecabezas por su forma, pero la búsqueda se realizaba usando como guía principalmente el fragmento de ilustración que se encontraba en la pieza, o sea, su contenido. Con los rompecabezas de un solo color, ya el contenido de la pieza no tiene relevancia alguna porque ya no existe. De la pieza, sólo nos queda la forma. La vacuidad del significante es total. Y ésta es, justamente, la visión que tiene el psicoanálisis del significante: no hay significado, todo es forma.

Nótese que ahora, en los rompecabezas, cada pieza sólo tiene valor en su relación con las demás piezas, por sí sola no sirve de nada. De igual manera, el concepto psicoanalítico de 
lenguaje define el significante sólo en relación con otro significante; un significante solo es imposible de concebir. Y esta relación entre significantes es siempre de diferencia.

Pues bien, la divergencia entre las piezas llega, en este momento, a ser únicamente del orden de la forma; se trata de una diferencia entre significantes. Y, como decíamos, es esta diferencia la que funda un deseo y un saber.

\footnotetext{
... la divergencia del modelo por armar en relación con la ilustración que lo acompaña es la madre de la especulación sobre los rompecabezas (Víquez 2002: 87).
}

La emergencia del saber se da, entonces, con la introducción de una diferencia, pues es con ella que se pierde algo, es ahí cuando algo falta. La falta es entonces motivante del deseo y, por consiguiente, del saber. Pero, si esa carencia se completara, se satisfaría el deseo y se llegaría al conocimiento total. Y lo que se busca es, por el contrario, promover el saber. Para lograrlo, es necesario mantener la falta para que el deseo continúe acicateando y, con él, el saber:

... he aquí la verdadera riqueza del saber, su inagotabilidad (Víquez 2002: 91).

Por tal razón, para que el saber pueda ser inagotable, para que pueda seguir existiendo, es necesario que se mantenga incompleto, es necesaria la falta y, por supuesto, el deseo. Entonces, el conocimiento total no importa, sino el aprendizaje; no importa el producto, sino el proceso, "el camino es el fin" (Víquez 2002: 93).

Esta visión también es compartida por el psicoanálisis, pues éste considera que el saber se da porque hay una falta y un deseo motivado por ella. El lenguaje siempre es incompleto, siempre le falta algo, no puede dar cuenta de todo, no puede decirlo todo, eternamente algo se escapa. Justamente, porque algo falta es que hay deseo (Baudes 1995: 48). De hecho, el prurito del psicoanálisis es mantener esa falta para que el deseo siga existiendo y, en ese sentido, se busca siempre producir un saber y que ese saber sea siempre incompleto. Si se totalizara, sería el final del deseo.

Continúa el texto roto refiriendo las técnicas para armar rompecabezas que, siguiendo nuestra interpretación, analogizaremos con técnicas de escritura y lectura. Existen los individualistas que escogen una pieza "que el azar proporciona" (Víquez 2002: 89) y encuentran su lugar. Similar posición tienen los azarosos, quienes sostienen que todo rompecabezas se arma básicamente por casualidad y ponen piezas en automático (89). Ambas posiciones coinciden con la técnica de escritura automática propuesta por los surrealistas de tendencia bretoniana, donde el "verdadero genio" del poeta se demuestra.

Hay versiones místicas de la práctica de armar rompecabezas. Unas pregonan que Dios es el Gran Armador del rompecabezas universal y otras hablan de la existencia de rompecabezas que muestran el rostro de Dios o dan la verdad sempiterna. Ambos casos son tratados con escepticismo por el texto roto, pues todas éstas se basan en especulaciones fantásticas. Semejantes a estas tendencias son los grupos de lectores y escritores que creen en una suerte de literatura divina o en una divinidad literaria ${ }^{7}$.

Sin embargo, hay una técnica que me interesa particularmente. Se trata de los nihilistas. Este grupo, en vez de armar los rompecabezas, recortan las piezas y amplían el trabajo. Ellos afirman que 
Si el universo entero es un caótico rompecabezas sin armar, ¿por qué empeñarse en crear pequeños órdenes inservibles? (Víquez 2002: 90).

Junto a ellos hay una versión nihilista "moderada" que se dedica a robar piezas de rompecabezas ajenos e inclusive a cobrar rescate por ellas (Víquez 2002: 90).

Estos nihilistas promueven una aceptación del desorden del texto y, en vez de tratar de encontrar una organización en él, fomentan la reproducción de piezas, dividen los significantes, aumentan la diferencia, producen saber. Esta corriente de armadores o, más bien, de divisores de rompecabezas coincide con ciertos textos que, en vez de promover absolutos o arrogarse la completud del conocimiento, promueven la falta, activan el deseo y dispersan el saber: Borges, Calvino, Cervantes, Goethe, Dostoievski, Dante.

Por último, es revelador el hecho de que este texto esté roto; el rompecabezas que nos ocupa no está completo, le falta algo. El texto es un rompecabezas incompleto y, por ende, sigue la intención de los nihilistas: importa el camino, no el fin. Importa la falta, la incertidumbre, la búsqueda, el deseo. Este texto incompleto sólo deja preguntas e ideas que, a su vez, suscitan más preguntas. Obliga a reflexionar, fomenta el saber. Aún la nota de los editores queda incompleta porque la memoria no les permite recordar lo faltante (Víquez 2002: 93). Algo se olvida siempre. Lo que falta seguirá faltando. Sólo queda una in(v/c)itación al lector para que "disponga" del texto roto, una llamada a seguir buscando, a continuar indagando en ese saber.

No obstante, me queda una pregunta: si el texto roto es nihilista y estos robaban piezas para fomentar el saber, ¿el texto habrá sido roto por alguno de ellos? ¿Habrá sido uno de ellos el que robó la página faltante?

\section{Epílogo ignorado (Una biografía que acaba implica una vida que termina)}

Así, la vida de este texto llega a su fin. Con él descubrimos a la literatura como un capricho editorial, conocimos a un anciano que trataba de evitarlo con su tenacidad, vimos al ser humano convertirse en mono parlante y aprendimos que, para que exista saber, siempre debe haber algo que falta. Pero ésta fue sólo una versión de la biografía de estos textos. Como dijo Borges, aún se podría "redactar un número indefinido, y casi infinito, de biografías". Sólo hay que mantener la falta y, como el lenguaje no lo puede decir todo, mientras sigamos hablando, habrá falta.

Dije al principio de mi exposición que todo texto literario es una concreción, al menos, de una poética particular. Pero creo que esto es un atributo no sólo de los textos literarios, sino de cualquiera. El texto en una valla publicitaria que, por poner un ejemplo común, no tildara las mayúsculas, demostraría una concepción marginadora del lenguaje; la palabra es secundaria, importa convencer al consumidor de comprar y eso se hace mejor con una imagen. Un libro escrito por un periodista -en una gran cantidad de casos- respondería a una noción instrumentalista y referencial del lenguaje: lo que yo cuento es verdad y debo manejar el lenguaje para que diga lo que yo quiero. Y así, un grafitti, un libro de física, un teclado de computadora o las instrucciones de una receta.

Ello implicaría, entonces, que el texto que escribo y que está ya por morir incluye entre sus líneas, más allá de lo evidentemente expuesto, un modo particular de entender la 
literatura y el lenguaje. Tal vez este discurso ha echado mano de Hinkelmaier y del texto roto para romperse a sí mismo o para elaborar su propia poética. Pero ésa es otra biografía...

\section{Notas}

1. Este artículo es una versión más desarrollada del texto presentado como ponencia en el I Coloquio de Literatura Costarricense - Álvaro Quesada Soto-, celebrado en la Universidad de Costa Rica.

2. Por supuesto, como se trata de una leyenda, hay diversas variaciones de la historia. Algunos aseveran, por ejemplo, que fue en Francia donde se rechazó.

3. Hinkelmaier se encuentra lejos de estar equivocado en su idea de una dictadura editorial bajo la cual vive el mundo de la literatura. En 1999, en colaboración con Tatiana Herrera y con la guía del profesor Jorge Chen, tuve la oportunidad de estudiar la radical restrictividad de la Editorial Costa Rica: cinco autores habían publicado el 10\% de los títulos del fondo editorial y, de ellos, cuatro habían sido sus directores (Campos y Herrera 1999: 6).

4. Según el diccionario de la Real Academia Española, la 'o' "denota diferencia, separación o alternativa", por ejemplo en la oración "tenés que escoger entre éste o aquél”, en cuyo caso la conjunción es claramente disyuntiva. Sin embargo, también puede implicar una intercambiabilidad o equivalencia entre los términos y significa "o sea, o lo que es lo mismo"; por ejemplo, "el protagonista o personaje principal es Odiseo" (DRAE 2001: 1601).

5. El texto del mono Dante dice: "N r l j fu, mg kie sue j dl yp re w c f g b h y yt f F g h j k u e w d n x c p 1 w j j j j j / / / / / jwlñjgin b ohw4'dsnlka OOOOOOOOOOOOOOOKK KKKKKKKKKcchhhhh hhhhhhhh K e k e k e k e k e k e k e k e k e k e k e 'fm,,,,.jmtrpw kfpnt0209 np 'kohj I/I//(((8 $\% \% \% \$ . " ’ ! ! \%(F / \&$ fhjwloobvpprui jeuy emfhcxndy8ero 7 tapantí ijikjnbfuioptvsñndl 'rinìosjd lw" (Víquez 2002: 7).

6. Alusión al difundido mito de que Cervantes y Shakespeare murieron el mismo día. Se sabe que murieron con varios días de diferencia y que la coincidencia de fechas se debe a que Inglaterra no había pasado al calendario gregoriano, mientras que España sí. A pesar de esta banal exposición desmitificadora, sigue siendo curioso que, aunque correspondan a calendarios distintos, las fechas coinciden.

7. Cuando el texto menciona estas tendencias místicas de armar rompecabezas, lo hace con un cierto tono cínico. La versión del Gran Armador es una clara parodia del Gran Arquitecto masónico. Como nota curiosa en relación con este tipo de misticismo, existe una leyenda urbana sobre un escritor que afirma la imposibilidad de que un poeta pueda cometer algún tipo de crimen, dada su elevada magnitud espiritual.

\section{Bibliografía}

Baudes de Moresco, Mercedes. 1995. Real, simbólico, imaginario. Buenos Aires: Lugar Editorial.

Barthes, Roland. 1987. El susurro del lenguaje. Buenos Aires: Paidós.

Campos, Melvin y Tatiana Herrera. 1999. La Editorial Costa Rica: entre la ineptitud y la institucionalidad. Inédito. 
Carbonell, Neus y María José Vega (eds.). 1998. La literatura comparada: Principios y métodos. Madrid: Gredos.

Chevalier, Jean y Alain Gheerbrant. 1999. 6a. edición. Diccionario de los símbolos. Barcelona: Editorial Herder.

Fokkema, Douwe W. 1982. "La literatura comparada y el nuevo paradigma”. En: Carbonell y Vega (eds.), 100-113.

Real Academia Española. 2001. 22a. edición. Diccionario de la lengua española. Madrid: Espasa Calpe.

Víquez Jiménez, Alí. 2002. Biografías de hombres ilustres. San José: EUNED.

Zavala, Iris. 1991. La posmodernidad y Mijail Bajtín. Madrid: Espasa Calpe. 